\title{
Plan de mejora de la investigación en la producción científica de los estudiantes de Ingeniería de Transportes de la Universidad Nacional Federico Villarreal
}

Improvement plan in the research in scientific production of Transport
Engineering students of Federico Villarreal National University

\author{
Violeta Romero Carrión ${ }^{\mathrm{I}}$ \\ Jorge Franco Medina ${ }^{\mathrm{I}}$ \\ Rosalvina CAmpos PÉrez ${ }^{I}$
}

\begin{abstract}
Resumen
Se presentan los efectos de la aplicación del Plan de Mejora de la Investigación en el incremento de la producción científica de los estudiantes de Ingeniería de Transportes. El Plan de Mejora de Investigación abordó la capacitación en uso de normas APA, elaboración de proyectos y redacción del informe final. Se elaboró el cuestionario de 20 preguntas y se aplicó al mismo grupo de 36 estudiantes, antes y después del tratamiento. Los resultados de la producción científica en las dimensiones, generación de proyectos, informe final y publicaciones evidencian una mejoría en todos los ítems del cuestionario, lo que corrobora la hipótesis del investigador que resultó altamente significativa y, en consecuencia, la aplicación del Plan de mejora de la Investigación incrementó la producción científica de los estudiantes de Ingeniería de Transportes.
\end{abstract}

Palabras clave: plan de mejora, investigación científica, producción científica

\begin{abstract}
In this research we present the effects of the implementation of the Research Improvement Plan on the increasing scientific production by Transport Engineering Students. The Research Improvement Plan addressed training in the use of APA standards, project development and drafting of the final report. The 20-question questionnaire was prepared and applied to the same group of 36 students, before and after treatment. The results of scientific production in the dimensions of project generation, final report and publications show an improvement in all items of the questionnaire, which validates the researcher's hypothesis, one that proved to be highly significant and, consequently, the implementation of this Research Improvement Plan increased the scientific production of Transport engineering students.
\end{abstract}

Key words: improvement plan, scientific research, scientific production

\footnotetext{
(C) Los autores. Este artículo es publicado por la Revista Campus de la Facultad de Ingeniería y Arquitectura de la Universidad de San Martín de Porres. Este artículo se distribuye en los términos de la Licencia Creative Commons Atribución No-comercial - Compartir-Igual 4.0 Internacional (https://creativecommons.org/licenses/ CC-BY), que permite el uso no comercial, distribución y reproducción en cualquier medio siempre que la obra original sea debidamente citada. Para uso comercial contactar a: revistacampus@usmp.pe.
} 


\section{INTRODUCCIÓN}

La Organización de las Naciones Unidas para la Educación, la Ciencia y la Cultura (UNESCO) en la Declaración Mundial sobre la Educación Superior en el siglo XXI (1998) sostiene lo siguiente:

La calidad de la enseñanza superior es un concepto pluridimensional que debería comprender todas sus funciones y actividades: enseñanza y programas académicos, investigación y becas, personal, estudiantes, edificios, instalaciones, equipamiento y servicios a la comunidad y al mundo universitario (art.11, 1998)

En el contexto de la autoevaluación, acreditación y el licenciamiento es imprescindible considerar tal concepto pluridimensional para aplicarlo bajo el enfoque sistémico y de la mejora continua (SINEACE, 2016). La calidad de enseñanza superior, también requiere de la internacionalización, la movilidad de profesores y estudiantes, las redes de investigadores y los proyectos de investigación internacionales.

Las tendencias en materia de investigación y desarrollo (I\&D) varían, considerablemente, en función de los países. En Japón, se ha fomentado la $I \& D$ en la investigación universitaria, mientras que en Rusia, ese mismo sector se ha reducido bruscamente con respecto al nivel del pasado. En Escandinavia y los Estados Unidos han permanecido estables, pero el apoyo del sector público ha disminuido en los países en que se ha reducido la función de financiación del gobierno (UNESCO, 2009).

Por otro lado, Frenzel \& Rojas (2007) determinaron el impacto de la evaluación y acreditación en el ámbito universitario de la Universidad Tecnológica Nacional de Tucumán - Argentina mediante el método analítico. Concluyen que la opinión de los docentes encuestados refleja que se han obtenido ciertos logros tras el proceso de acreditación como en el desempeño docente, en la gestión de las autoridades, mejoras en biblioteca y laboratorios. En cuanto a la investigación, el impacto sobre el incremento en la cantidad de proyectos institucionales $y$ de carrera, de docentes y investigadores y el número de trabajos presentados en eventos científicos es de un nivel alto para el $30 \%$ y de un nivel medio para el $40 \%$.

En ese contexto, quizás uno de los logros mayores de este proceso es el haber creado una política de calidad en las instituciones, que se ha traducido en un incremento del grado de responsabilidad de todos los actores involucrados para generar procesos continuos de superación y mejoramiento. Zapata (2012), director del Proyecto del Plan de mejoramiento y fortalecimiento del Programa de Formación de investigadores educacionales con estándar internacional de la Pontificia Universidad Católica de Chile (PUC), sostiene que mejorar la calidad e impacto de la investigación educacional producida en los distintos centros y universidades del país es un área crítica.

El Doctorado de Educación de la PUC ha avanzado en estos últimos años en materia de organización curricular y renovación de planta académica. Como prueba de ello, es el primer programa en su área acreditado en el país. En este escenario tres aspectos críticos requieren ser abordados: primero, 
internacionalizar las experiencias $y$ actividades formativas.

Los investigadores educacionales formados en Chile requieren conectarse de primera fuente con la producción académica que toma lugar en los mejores centros académicos del mundo. Segundo, fortalecer en términos curriculares competencias de investigación que impacten en la calidad de la producción académica de los estudiantes y futuros doctores. Tercero, lo que también constituye un patrón internacional, el programa posee una limitada conexión con los problemas prácticos de aula y gestión directiva en escuelas y pone mayor énfasis teórico en desconexión con la práctica. Un objetivo, aquí es entonces, crear mecanismos para fortalecer la conexión durante la formación doctoral.

Sweden (2013) reporta que la educación superior en Suecia se caracteriza por la manera en que los estudiantes asumen la responsabilidad de sus propios estudios, y por la relación relajada e informal con los profesores, bajo el lema "libertad con responsabilidad". El sistema sueco de educación superior ha cambiado, significativamente, durante los últimos años como resultado de lo que se conoce como el Proceso de Bolonia de 1999, cuyo objetivo es crear un Espacio Europeo de Educación Superior (EEES), cuyos objetivos son: promover la movilidad, la empleabilidad y la competitividad de Europa como un continente de la educación.

Piscoya (2008) refiere que el estado peruano es el que menos invierte en América Latina como porcentaje de su PBI en educación superior, bibliotecas y en lo que se denomina investigación y desarrollo. Ciertamente, con estos condicionantes de política nacional resulta muy comprensible que las universidades peruanas tanto públicas como privadas se dediquen, casi exclusivamente, a la formación profesional. Existe un precario posicionamiento de nuestras universidades, dentro de la comunidad internacional, como productoras de ciencia y tecnología.

William Sánchez, Director de Investigación y Estudios del Consejo Nacional de Ciencia y Tecnología (CONCYTEC), indicó que el Perú solo gasta el $0.08 \%$ del Producto Bruto Interno (PBI) en investigación y desarrollo, siendo esta cifra muy inferior a casos como el de Colombia (0.25\%), Chile $(0.38 \%)$ y México $(0.54 \%)$ por citar algunos ejemplos (CONCYTEC, 2017).

Bermúdez (2009) sostiene que, en el Perú, la universidad no se concibe como una institución de gran nivel académico, productora de ciencia, tecnología e innovación, sino considerada como formadora de profesionales. Por tal razón, la gran mayoría de universidades del Perú no produce ciencia y tecnología en los niveles de calidad que los tiempos actuales demandan. Por otro lado, lo poco investigado y producido en algunas facultades no está debidamente divulgado ni siquiera en sus propios ámbitos porque no existen mecanismos de difusión como las revistas científicas ya sean impresas o virtuales, que difundan los productos de las investigaciones. Si los resultados de las investigaciones no se publican o no se divulgan para conocimiento, análisis, validación o refutación en el ámbito de la comunidad científica, es como si no se hubieran realizado. 
La Universidad Nacional Mayor de San Marcos (UNMSM) en el año 2013 en su Plan Estratégico Institucional (PEI) 2012 - 2021 considera en su Modelo Educativo dos aspectos que se condicen con los estándares de calidad: Investigación formativa (como eje transversal del currículo) y Difusión de la producción intelectual y científica (Promueve y propicia la publicación de las actividades de la comunidad académica con visualización en internet y en revistas especializadas e indizadas). Se aprecia que en la elaboración del PEI ya se toma en cuenta lo que se evaluará para los fines de la Acreditación. Es un proceso inicial que esperamos se consolide en la medida que se ejecute en el Plan Operativo Institucional. En el año 2015, la Universidad Nacional Federico Villarreal (UNFV) considera en el primer artículo de su estatuto como misión esencial la formación profesional, la investigación, la creación del conocimiento científico, tecnológico y universal y pone énfasis en la investigación a desarrollar por los estudiantes y docentes.

El Sistema Nacional de Evaluación, Acreditación y Certificación de la Calidad Educativa (SINEACE) es un organismo técnico especializado adscrito al Ministerio de Educación. Fue creado en el 2006 a través de la Ley No28740. $\mathrm{Su}$ finalidad es garantizar a la sociedad que las instituciones educativas públicas y privadas ofrezcan un servicio de calidad, y que los trabajadores peruanos estén altamente calificados para la labor que realizan. En el reglamento de la Ley del SINEACE (2007) se establece que la Autoevaluación contempla la elaboración del Informe Final de autoevaluación, el cual es el resultado del mismo proceso que detalla de manera concisa, organizada y clara el desarrollo y sus resultados, así como el Plan de Mejora correspondiente, acompańado de las respectivas fuentes de verificación que sustentan la justificación de cumplimiento del estándar.

La presente investigación contribuye con el Plan de Mejora de la investigación, conducentes al incremento de la producción científica entendiéndose como tal la visibilización de los resultados de las investigaciones realizadas por los estudiantes del nivel superior en el marco de la acreditación de las carreras profesionales y el licenciamiento. Es por ello, que consideramos importante la elaboración de un Plan de Mejora de la Investigación que fue aplicado a los estudiantes con el propósito de generar proyectos de investigación direccionados a la realización de sus tesis para la titulación, producción de artículos y publicaciones considerando el enfoque sistémico y la mejora continua.

\section{Método}

Nivel de la Investigación: Explicativa (causa - efecto)

Diseño: Cuasi experimental

$$
\begin{array}{llll}
\mathbf{G} & \mathrm{O}_{1} & \mathrm{X} & \mathrm{O}_{2}
\end{array}
$$

G: Grupo (alumnos antes y después del experimento)

$\mathrm{O}_{1}$ : Observación inicial (pre-prueba)

$\mathrm{X}$ : Tratamiento experimental (aplicación del plan de mejora de la investigación)

$\mathrm{O}_{2}$ : Observación final (pos-prueba luego del tratamiento)

\section{Población}

$\mathrm{N}=100$ estudiantes de 2 do año de la Escuela Profesional de Ingeniería de Transportes, FIIS - UNFV 


\section{Muestra}

Seleccionamos de forma directa e intencionadamente a los individuos de la población. Tamaño de muestra: $\mathrm{n}=36$ alumnos de 2 do año de la EPIT - FIIS

\begin{tabular}{ccc} 
Varones & Mujeres & TOTAL \\
\hline 23 & 13 & 36
\end{tabular}

La encuesta fue aplicada, de manera presencial, a los estudiantes de 2 do año de Ingeniería de Transportes. El instrumento aplicado fue un cuestionario de 20 preguntas, el cual contempla tres aspectos: generación de proyectos, ejecución de proyectos y difusión. Posee un grado de confiabilidad altamente significativa $(\alpha=0,917)$.

La validez se efectuó a juicio de experto. Se consideró a seis especialistas dedicados a la investigación y docencia quienes estuvieron de acuerdo en que el cuestionario medía de manera pertinente los contenidos expresados en cada uno de los ítems.

\section{Procedimiento}

- Se aplicaron los cuestionarios seleccionados.

- Se utilizaron tablas y figuras para mostrar los datos de la investigación mediante el uso del paquete estadístico SPSS 21.0. Luego se procedió al procesamiento de la información con el reporte estadístico utilizando para ello diversos estadígrafos.

- Se aplicó T de Student para las muestras independientes.

- Criterio de decisión: A un nivel de confianza de $95 \%$ si "p" es menor que 0,05 se rechaza $\mathrm{H}_{0}$.

\section{Resultados}

Para la variable independiente: Plan de mejora de la investigación

Tabla 1

Plan de Mejora de la Investigación en la Producción Cientifica-EPIT

\begin{tabular}{|c|c|c|c|c|c|c|c|}
\hline $\mathbf{N}^{\circ}$ & Actividades & Responsables & Meta & Recursos & $\begin{array}{l}\text { Fecha } \\
\text { límite }\end{array}$ & $\begin{array}{l}\text { Indicador de } \\
\text { cumplimiento }\end{array}$ & Eval. \\
\hline 1 & \begin{tabular}{ll}
\multicolumn{2}{l}{ Capacitación } \\
en el uso de \\
las normas \\
APA
\end{tabular} & $\begin{array}{l}\text { Docente de } \\
\text { Metodología } \\
\text { de la } \\
\text { Investigación }\end{array}$ & $\begin{array}{c}36 \\
\text { alumnos }\end{array}$ & $\begin{array}{l}\text { - } \text { Normas } \\
\text { - Multimedia } \\
\text { - Internet }\end{array}$ & $\begin{array}{c}\text { Diciembre } \\
2015\end{array}$ & $\begin{array}{l}\text { - Redacción de } \\
\text { referencias } \\
\text { - Citas de } \\
\text { autores }\end{array}$ & \\
\hline 2 & $\begin{array}{l}\text { Capacitación } \\
\text { e n } \\
\text { elaboración } \\
\text { de proyectos }\end{array}$ & $\begin{array}{l}\text { Docente de } \\
\text { Metodología } \\
\text { de la } \\
\text { Investigación }\end{array}$ & $\begin{array}{c}36 \\
\text { alumnos }\end{array}$ & $\begin{array}{l}\text { - Instrumentos } \\
\text { de medición } \\
\text { - Multimedia } \\
\text { - Internet }\end{array}$ & $\begin{array}{l}\text { Julio } \\
2016\end{array}$ & 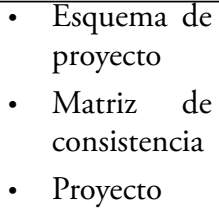 & \\
\hline 3 & $\begin{array}{l}\text { Capacitación } \\
\text { M a n e j o } \\
\text { SPSS } \\
\text { Redacción } \\
\text { informe de } \\
\text { investigación }\end{array}$ & $\begin{array}{l}\text { Docente de } \\
\text { Metodología } \\
\text { de la } \\
\text { Investigación }\end{array}$ & $\begin{array}{c}36 \\
\text { alumnos }\end{array}$ & $\begin{array}{ll}\text { - } & \text { PC y SPSS } \\
\text { - } & \text { Multimedia } \\
\text { - } & \text { Internet } \\
\text { - } & \text { Auditorio } \\
\text { - } & \text { Posters }\end{array}$ & $\begin{array}{c}\text { Diciembre } \\
2016\end{array}$ & 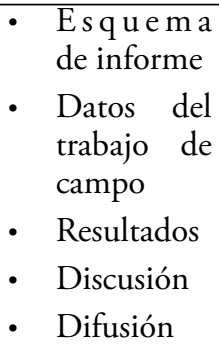 & \\
\hline
\end{tabular}


Para la variable dependiente: Producción Científica

Las Figuras 1, 2 y 3 muestran los ítems agrupados en función a las tres dimensiones consideradas en el cuestionario que corresponden a su vez a la variable dependiente Producción Científica, antes y después del tratamiento Plan de Mejora de la Investigación. Dichas dimensiones son las siguientes:

- Generación de proyectos

- Difusión del informe final o resultados

- Publicaciones

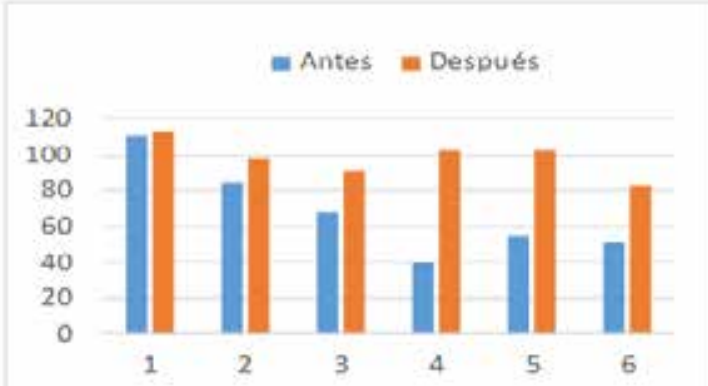

Figura 1. Dimensión: Generación de proyectos

En la Figura 1 se observa que la mayor variación por efecto del tratamiento, se registra para la pregunta cuatro del cuestionario, "En qué medida conoce las normas APA para elaborar las referencias".

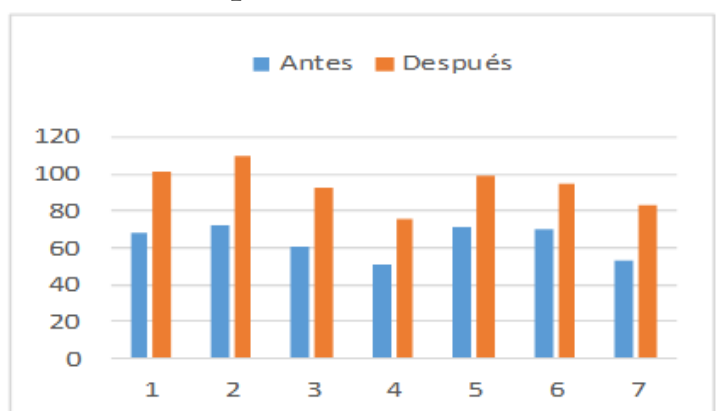

Figura 2. Dimensión: Informe final

En la Figura 2 se observa que la mayor variación favorable se registra para el caso (2) de la pregunta del cuestionario, "Grado en que diferencia una tabla de una figura, para mostrar los datos recogidos".

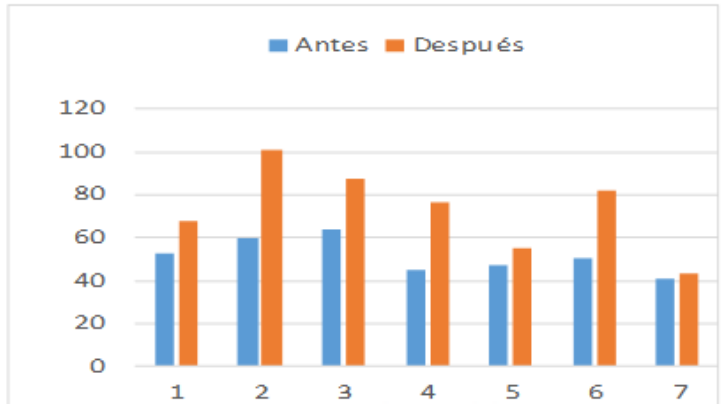

Figura 3. Dimensión: Publicaciones

En la Figura 3, se observa que la mayor variación favorable se registra para el caso (2) de la pregunta del cuestionario "El grado de conocimiento para redactar el abstract de una investigación". Cabe señalar que, en virtud a la aplicación del Plan de Mejora de la Investigación de este trabajo se han mostrado algunas evidencias del incremento de la producción científica en la Escuela Profesional de Ingeniería de Transportes de la Facultad de Ingeniería Industrial y de Sistemas de la Universidad Nacional Federico Villarreal como consta en la Memoria del Instituto de Investigación de la FIIS 2015 -2016. Se lograron los siguientes resultados:

- Presentación de un promedio de 15 Proyectos de Investigación en las diversas asignaturas de Metodología de la Investigación que han posibilitado una mayor participación en las Jornadas Científicas Inter facultades de la UNFV en los años 2015 y 2016.

- Asistencia a eventos de carácter científico organizados en la Facultad $y$ en otras instituciones: Taller uso de Base de datos ProQuest, Curso de SPSS para análisis de datos, Conferencia sobre Simbiosis entre la ciencia y la tecnología, Exposición de los trabajos de investigación de los docentes.

- Participación de estudiantes de la Escuela Profesional de Ingeniería 
de Transportes en congresos internacionales como asistentes $y$ ponentes.

\section{Prueba de Hipótesis}

Antes de aplicar el estadístico $\mathrm{T}$ de Student se realizó la prueba de normalidad y eligió Shapiro - Wilk debido a que el número de datos es menor a 50 .
Prueba de hipótesis para la normalidad

$\mathrm{H}_{0:}$ La variable producción científica tiene distribución normal.

$\mathrm{H}_{1:}$ La variable producción científica no tiene distribución normal.

Criterio de decisión: Si $\mathrm{p}<0,05$ se rechaza la hipótesis nula.

Tabla 2

Pruebas de normalidad

\begin{tabular}{|c|c|c|c|c|c|c|}
\hline & \multicolumn{4}{|c|}{ Kolmogorov-Smirnor ${ }^{2}$} & \multicolumn{3}{c|}{ Shapiro-Wilk } \\
\cline { 2 - 7 } & Estadístico & $\mathrm{gl}$ & Sig. & Estadístico & gl & Sig. \\
\hline P.C. Después &, 119 & 20 &, $200^{*}$ &, 929 & 20 &, 148 \\
\hline
\end{tabular}

* Esto es un límite inferior a la significación verdadera.

${ }^{a}$ Corrección de significación de Lilliefors.

Como se muestra en la Tabla 2, la variable Producción Científica después del tratamiento $(\mathrm{p}=0,148)$ resulta mayor que $(0,05)$. En consecuencia, no se puede rechazar la hipótesis nula y se concluye que la variable producción científica tiene distribución normal.

\section{Prueba de hipótesis específicas:}

1) Se aplicó $T$ de Student para muestras relacionadas generación de proyectos de la variable dependiente, tanto antes como después del tratamiento "Aplicación del Plan de Mejora”.

Tabla 3

Prueba T de Student de muestras emparejadas: Generación de proyectos

\begin{tabular}{|c|c|c|c|c|c|c|c|c|}
\hline & \multicolumn{5}{|c|}{ Diferencias emparejadas } & \multirow[b]{3}{*}{$\mathrm{t}$} & \multirow[b]{3}{*}{$\mathrm{gl}$} & \multirow{3}{*}{$\begin{array}{c}\text { Sig. } \\
\text { (bilateral) }\end{array}$} \\
\hline & \multirow[b]{2}{*}{ Media } & \multirow{2}{*}{$\begin{array}{c}\text { Desviación } \\
\text { estándar }\end{array}$} & \multirow{2}{*}{$\begin{array}{c}\text { Media de } \\
\text { error } \\
\text { estándar }\end{array}$} & \multicolumn{2}{|c|}{$\begin{array}{c}95 \% \text { de intervalo } \\
\text { de confianza de la } \\
\text { diferencia }\end{array}$} & & & \\
\hline & & & & Inferior & Superior & & & \\
\hline $\begin{array}{l}\text { Dimensión } 1 \\
\text { Antes-Después }\end{array}$ & $-30,500$ & 22,134 & 9,036 & $-53,728$ & $-7,272$ & $-3,37$ & 5 & \\
\hline
\end{tabular}

$\mathrm{H}_{1}$ : La aplicación del Plan de Mejora de la Investigación incrementa la generación de proyectos de los estudiantes de Ingeniería de Transportes UNFV.
$\mathrm{H}_{0}$ : La aplicación del Plan de Mejora de la Investigación no incrementa la generación de proyectos de los estudiantes de Ingeniería de Transportes UNFV. 


\begin{tabular}{|l|}
\hline $\mathrm{p}=0,020 \rightarrow \mathrm{p}<0,05 \rightarrow$ se rechaza $\mathrm{H}_{0}$ \\
\hline Se acepta $\mathrm{H}_{1}$ \\
La aplicación del Plan de Mejora de la Investigación incrementa la generación \\
de proyectos de los estudiantes de Ingeniería de Transportes UNFV.
\end{tabular}

2) Se aplicó la $T$ de Student para muestras relacionadas a la dimensión 2 Difusión informe final de la variable dependiente, tanto antes como después del tratamiento "Aplicación del Plan de mejora”.

$\mathrm{H}_{1}$ : La aplicación del Plan de Mejora de la Investigación incrementa la difusión de los resultados de investigación en eventos donde participan los estudiantes de Ingeniería de Transportes UNFV.

$\mathrm{H}_{0}$ : La aplicación del Plan de Mejora de la Investigación no incrementa la difusión de los resultados de investigación en eventos donde participan los estudiantes de Ingeniería de Transportes UNFV.

Tabla 4

Prueba T de Student de muestras emparejadas: Difusión de resultados - Informe Final

\begin{tabular}{|c|c|c|c|c|c|c|c|c|}
\hline & \multicolumn{5}{|c|}{ Diferencias emparejadas } & \multirow[b]{3}{*}{$\mathrm{t}$} & \multirow[b]{3}{*}{$\mathrm{gl}$} & \multirow{3}{*}{$\begin{array}{c}\text { Sig. } \\
\text { (bilateral) }\end{array}$} \\
\hline & \multirow[b]{2}{*}{ Media } & \multirow{2}{*}{$\begin{array}{l}\text { Desviación } \\
\text { estándar }\end{array}$} & \multirow{2}{*}{$\begin{array}{c}\text { Media de } \\
\text { error } \\
\text { estándar }\end{array}$} & \multicolumn{2}{|c|}{$\begin{array}{c}95 \% \text { de intervalo } \\
\text { de confianza de la } \\
\text { diferencia }\end{array}$} & & & \\
\hline & & & & Inferior & Superior & & & \\
\hline $\begin{array}{c}\text { Dimensión } 2 \\
\text { Antes-Después }\end{array}$ & $-30,143$ & 4,670 & 1,765 & $-34,462$ & $-25,824$ & 17.07 & 6 & \\
\hline
\end{tabular}

\begin{tabular}{l}
$\qquad \mathrm{p}=0,000 \rightarrow \mathrm{p}<0,05 \rightarrow$ se rechaza $\mathrm{H}_{0}$ \\
\hline Se acepta $\mathrm{H}_{1}$ \\
La aplicación del Plan de Mejora de la Investigación incrementa la difusión de \\
los resultados en eventos donde participan estudiantes de Ingeniería de \\
Transportes UNFV.
\end{tabular}

3) Se aplicó $\mathrm{T}$ de Student para muestras relacionadas a la dimensión 3, generación de publicaciones periódicas de la variable dependiente, tanto antes como después del tratamiento "Aplicación del Plan de mejora".

$\mathrm{H}_{1}$ : La aplicación del Plan de Mejora de la Investigación incrementa la generación de publicaciones periódicas de los estudiantes de Ingeniería de Transportes UNFV

$\mathrm{H}_{0}$ : La aplicación del Plan de Mejora de la Investigación no incrementa la generación de publicaciones periódicas de los estudiantes de Ingeniería de Transportes. 
Tabla 5

Prueba T de Student muestras emparejadas: Generación de publicaciones

Prueba de hipótesis general:

\begin{tabular}{|c|c|c|c|c|c|c|c|c|}
\hline & \multicolumn{5}{|c|}{ Diferencias emparejadas } & \multirow[b]{3}{*}{$\mathrm{t}$} & \multirow[b]{3}{*}{$\mathrm{gl}$} & \multirow{3}{*}{$\begin{array}{c}\text { Sig. } \\
\text { (bilateral) }\end{array}$} \\
\hline & \multirow[b]{2}{*}{ Media } & \multirow{2}{*}{$\begin{array}{l}\text { Desviación } \\
\text { estándar }\end{array}$} & \multirow{2}{*}{$\begin{array}{c}\text { Media de } \\
\text { error } \\
\text { estándar }\end{array}$} & \multicolumn{2}{|c|}{\begin{tabular}{|c|}
$95 \%$ de intervalo \\
de confianza de la \\
diferencia
\end{tabular}} & & & \\
\hline & & & & Inferior & Superior & & & \\
\hline $\begin{array}{c}\text { Dimensión } 3 \\
\text { Antes-Después }\end{array}$ & $-21,714$ & 14,068 & 5,317 & $-34,725$ & $-8,704$ & $-4,08$ & 6 & \\
\hline
\end{tabular}

\begin{tabular}{|l|}
\hline $\mathrm{p}=0,006 \rightarrow \mathrm{p}<0,05 \rightarrow$ se rechaza $\mathrm{H}_{0}$ \\
\hline Se acepta $\mathrm{H}_{1}$ \\
La aplicación del Plan de Mejora de la Investigación incrementa la generación \\
de publicaciones periódicas de los estudiantes de Ingeniería de Transportes \\
UNFV 2014-2016.
\end{tabular}

Se aplicó T de Student para muestras relacionadas de manera global a la variable dependiente "Producción científica" tanto antes como después del tratamiento "Aplicación del Plan de mejora".

$\mathrm{H}_{1}$ : La aplicación del Plan de Mejora de la Investigación incrementa la Producción Científica de los estudiantes de 2 do año de Ingeniería de Transportes UNFV.
$\mathrm{H}_{0}$ : La aplicación del Plan de Mejora de la investigación no incrementa la Producción Científica de los estudiantes de 2 do año de Ingeniería de Transportes UNFV.

\section{Criterio de decisión:}

Si la significancia ( $\mathrm{p}$ valor) $\mathrm{p}<0,05$ se rechaza la hipótesis nula.

Luego:

\begin{tabular}{|l|c|}
\hline \multicolumn{2}{|c|}{ Aplicando la prueba de hipótesis: } \\
\hline $\mathrm{p}=0,000 \rightarrow \mathrm{p}<0,05$ & Entonces se rechaza $\mathrm{H}_{0}$ \\
\hline Se acepta la $\mathrm{H}_{1}$ \\
La aplicación del Plan de Mejora de la Investigación incrementa la Producción \\
Científica de los estudiantes de 2do ańo de Ingeniería de Transportes UNFV. \\
\hline
\end{tabular}

\section{Discusión}

Se demuestra un nivel altamente significativo $(p=0,000)$ en base al estadístico de prueba ya que la aplicación del Plan de Mejora de la investigación incrementó la Producción Científica de los estudiantes de 2 do año de Ingeniería de Transportes.

Se corroboró dicho incremento de la producción científica, mediante algunas evidencias como: logro de presentación de un promedio de 15 Proyectos de 
Investigación en la Escuela de Ingeniería de Transportes, los cuales posibilitaron mayor participación en las Jornadas Científicas y asistencia a congresos internacionales. Lo que coincide con Frenzel \& Rojas (2007) que manifiestan el impacto en el incremento de proyectos, número de publicaciones y de trabajos presentados en eventos científicos por los estudiantes. Esto refleja que la aplicación del Plan de mejora de la Investigación muestra su bondad.

\section{Conclusiones}

La aplicación del Plan de Mejora de la Investigación en cada una de las tres dimensiones incrementó, en un nivel significativo $(\mathrm{p}=0,020)$ la generación de proyectos de investigación. También aumentó en un nivel altamente significativo $(\mathrm{p}=0,000)$ la difusión de los resultados de la investigación en eventos, $y$ en un nivel altamente significativo ( $p$ $=0,006$ ) la generación de publicación periódica mediante pósteres. Estas son evidencias registradas antes y después del tratamiento. Finalmente, a un nivel de confianza del $95 \%$ podemos afirmar que la aplicación del Plan de Mejora de la investigación, incrementó en un nivel altamente significativo $(\mathrm{p}=0,000)$ la Producción Científica de los estudiantes de 2 do año de Ingeniería de Transportes de la Universidad Nacional Federico Villarreal.

\section{Referencias}

Bermúdez, J. (2009). Investigación cientifica en el Perú: factor critico de éxito para el desarrollo del pais. España: Escuela de Negocios EOI.

CONCYTEC (2017). I Censo nacional de investigación y desarrollo a centros de investigación 2016

Frenzel \& Rojas (2007). Impacto de la evaluación y acreditación en el ámbito universitario de la Universidad Tecnológica de Nacional de Tucumán. Argentina.

Piscoya, L. (2008). Formación Universitaria Vs. Mercado Laboral II. Lima: ANR.

UNESCO (1998). Declaración Mundial sobre la Educación Superior en el siglo XXI. Art. 11.
UNESCO a (2009). Fórum sobre la enseñanza superior, la investigación y el conocimiento Paris.

UNESCO b (2009). Forum on Higher Education, Research and Knowledge from 2001 to 2009. Paris.

Universidad Nacional Federico Villarreal. (2015). Estatuto 2015 y modificaciones. Lima.

http://web2.unfv.edu.pe/sitio/ transparencia/transparenciauniversitaria/documentos-degestion-y-reglamentos/estatuto-dela-universidad

Universidad Nacional Mayor de San Marcos. (2012). Plan Estratégico 2012.Lima.

SINEACE (2016). Modelo de Acreditación para Programas de 
PLAN DE MEJORA DE LA INVESTIGACIÓN EN LA PRODUCCiÓN CIENTÍfICA DE LOS ESTUdiANTES DE INGENIERÍA DE Transportes de la Universidad Nacional Federico Villarreal

Estudios de Educación Superior Universitaria. https://www. sineace.gob.pe/wp-content/ uploads/2014/08/Anexo-1nuevo-modelo-programasResolucion-175.pdf

Sweden, S. (2013). La educación Superior e investigación en Suecia. Leído 19-09-16
http://www.sweden.se/sp/Inicio/ Educacion/Datos/Educacionsuperior-e-investigacion-en-Suecia/

Zapata, G. (2012). Plan de mejoramiento $y$ fortalecimiento del Programa de Formación de Investigadores educacionales con estándar internacional. Chile: Pontificia Universidad Católica de Chile. 
